\title{
Mediastinitis and pseudoaneurysm of brachiocephalic artery long after the resection of invasive thymoma and postoperative irradiation
}

\author{
Kenichi Okubo, MD, Jun Isobe, MD, Jiro Kitamura, MD, and Yoichiro Ueno, MD, Gifu, Japan
}

$\mathrm{M}$

ediastinitis is a complication after median sternotomy. We report a rare case of mediastinitis and mycotic pseudoaneurysm of the brachiocephalic artery that occurred long after the resection of invasive thymoma and postoperative irradiation and was treated with extensive procedures.

\section{Clinical Summary}

A 68-year-old woman was seen with skin ulceration and suppuration from the wound in the anterior chest wall. She had undergone resection of invasive thymoma through median sternotomy 65 months previously. This operative treatment included combined resection of pericardium, right lung, and superior vena cava, along with interposition of an artificial polytetrafluoroethylene graft between the brachiocephalic vein and the right atrium. The patient received adjuvant radiation therapy of $50 \mathrm{~Gy}$ to the mediastinum for pathologically diagnosed Masaoka stage III thymoma. Computed tomography of the chest showed a dilated branch of the aortic arch compressing the sternum posteriorly, an occluded artificial graft between the brachiocephalic vein and the right atrium, and low-density areas in the mediastinum (Figure 1). A culture of the suppuration grew Pseudomonas aeruginosa. Arteriography showed a sacral aneurysm of the brachiocephalic artery (Figure 2). The patient underwent excision of the aneurysm through median resternotomy and extended right collar incision for the diagnosis of mediastinitis and mycotic aneurysm of brachiocephalic artery. The implanted polytetrafluoroethylene graft was found to be filled with pus and was removed. After the sternum had been released from the aneurysm, the wall of which was found to be extremely thin, the aneurysm ruptured suddenly. Cardiopulmonary bypass was quickly instituted through the previously exposed cannulations of the femoral artery and vein, with the ruptured aneurysm compressed by the surgeon's finger. Selective cerebral perfusion was established with additional cannulations of the right axillary artery and the right atrium. The aneurysm of the brachiocephalic artery was excised under conditions of circulatory arrest with profound hypothermia. A Dacron polyester fabric graft was anastomosed

\footnotetext{
From the General Thoracic Surgery and Cardiac Surgery, National Hospital Organization, Nagara Medical Center, Gifu, Japan.

Received for publication March 14, 2005; accepted for publication May 3, 2005.

Address for reprints: Kenichi Okubo, MD, General Thoracic Surgery, National Hospital Organization, Nagara Medical Center, 1300-7 Nagara, Gifu 502-0071, Japan (E-mail: okubo@nagara-lan.hosp.go.jp).

J Thorac Cardiovasc Surg 2005;130:918-9

$0022-5223 / \$ 30.00$

Copyright $\odot 2005$ by The American Association for Thoracic Surgery doi:10.1016/j.jtcvs.2005.05.005
}

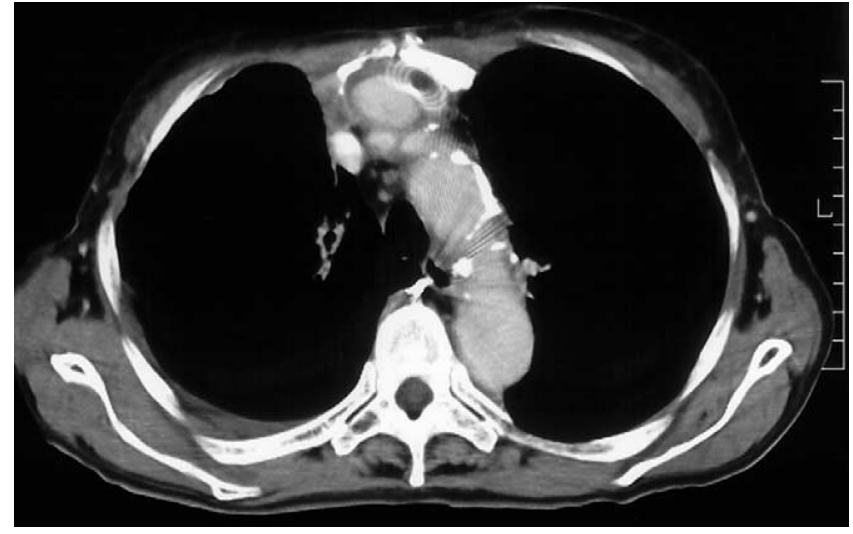

Figure 1. Chest computed tomography showing a dilated aortic branch compressing the sternum and an occluded artificial graft.

with the tailored aortic arch at the origin of the brachiocephalic artery proximally. The distal anastomosis was at the bifurcation of common carotid artery and subclavian artery, with cardiopulmonary bypass restarted without axillary artery perfusion. Circulatory arrest time was 22 minutes, and the duration of cardiopulmonary bypass was 224 minutes. After the patient was weaned from cardiopulmonary bypass, necrotic sternum was resected and the infected mediastinum was irrigated with povidone iodine. Pedicled

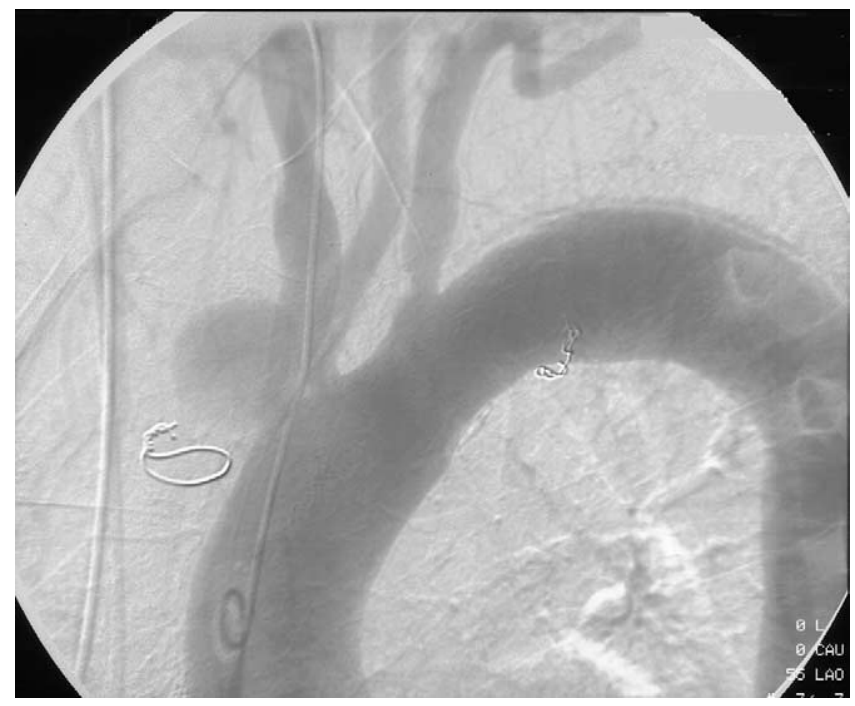

Figure 2. Arteriography showing a sacral aneurysm of brachiocephalic artery. 
omentum was then transposed to cover the mediastinum and the Dacron graft through the extended upper abdominal approach. The skin and subcutaneous tissues were resutured, with the defect in the sternum left. After the operation, the patient required mechanical ventilation for respiratory failure for 8 days. After endotracheal extubation, she recovered well. She has been doing well for 28 months after the operation.

\section{Comment}

Pseudoaneurysm of the brachiocephalic artery has been reported from a variety of etiologies. ${ }^{1}$ Traumatic injuries are the most frequently reported cause of this pseudoaneurysm. Blunt or stabbing injury ${ }^{2,3}$ and medically introduced trauma, ${ }^{4}$ such as catheterization, intravenous cannulation, and stenting, have occasionally been reported as causes. Infectious cause has infrequently been seen after cardiac surgery. ${ }^{5}$ Cannulation sutures or artificial grafts have become the foci of mediastinitis and have caused mycotic pseudoaneurysm of adjacent arteries.

Our patient showed mediastinitis and pseudoaneurysm of the brachiocephalic artery long after the surgical treatment for invasive thymoma. Because of the extent of the mediastinitis and the pus formation in the implanted graft, the pseudoaneurysm of the brachiocephalic artery appeared to be of infectious origin. The chronic mediastinitis was associated with previous median sternotomy and postoperative irradiation, although more than 5 years had passed since the resection of invasive thymoma. Adjuvant irradiation would have caused poor vascularization in the postoperative mediastinum, with consequent failure of protection against transsternal infection. Such a mycotic pseudoaneurysm of brachiocephalic artery after combined surgery and irradiation has seldom been reported in the literature. Extensive procedures-including removal of infective foci, necrotic sternum, and artificial graft; excision of the pseudoaneurysm; surgical irrigation; and omentum transposition-appear to have been successful in treating this extended mediastinal infection.

\section{References}

1. Kieffer E, Chieche L, Koskas F, Bahnini A. Aneurysms of the innominate artery: surgical treatment of 27 patients. J Vasc Surg. 2001;34: 222-8.

2. de Jose Maria B, Gomar C, Mestres C, Sorribes V, Moral V, Sala X. Pseudoaneurysm of the brachiocephalic artery caused by blunt chest trauma. J Thorac Cardiovasc Surg. 1995;110:863-5.

3. Reddi AA, Munasur MM, Naidoo RR, Steer DD. Traumatic innominate artery aneurysm 26 years after stab injury. Ann Thorac Surg. 2005;79: 1034-6.

4. Shield CF, Richardson JD, Buckley CJ, Hagood CO Jr. Pseudoaneurysm of the brachiocephalic arteries: a complication of percutaneous internal jugular vein catheterization. Surgery. 1975;78:190-4.

5. Katsumata T, Moorjani N, Vaccari G, Westaby S. Mediastinal false aneurysm after thoracic aortic surgery. Ann Thorac Surg. 2000;70: $547-52$.

\title{
Pulmonary segmental venous infarction after living- donor lobar lung transplantation
}

\author{
Cliff K. Choong, FRACS, Ding Z. Hu, MD, and Charles B. Huddleston, MD, St Louis, Mo
}

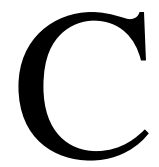

omplete obstruction of one or more of the major pulmonary veins after lung transplantation is a devastating complication. It results in hemorrhagic infarction of the affected lung within 4 to 6 hours, leading to irreversible lung damage. Reported treatments have included resection of the affected lobe and retransplantation. ${ }^{1,2}$

\footnotetext{
From the Division of Cardiothoracic Surgery, Department of Surgery, Washington University School of Medicine, St Louis, Mo.

Received for publication Jan 29, 2005; accepted for publication March 14, 2005.

Address for reprints: Charles B. Huddleston, MD, Professor of Surgery, No. 1 Children's Place, Suite 5S 50, Children's Hospital, St Louis, MO 63110 (E-mail: huddlestonc@msnotes.wustl.edu).

J Thorac Cardiovasc Surg 2005;130:919-21

$0022-5223 / \$ 30.00$

Copyright $\odot 2005$ by The American Association for Thoracic Surgery

doi:10.1016/j.jtcvs.2005.03.045
}

We present a case of pulmonary venous infarction after transplantation that was treated conservatively.

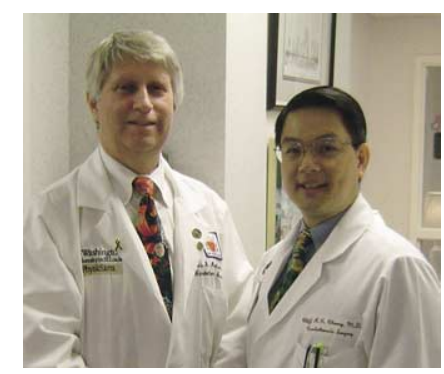

Huddleston and Choong (left to right)

\section{Clinical Summary}

A 16-year-old girl with a history of cystic fibrosis, multiple pulmonary infections, insulin-dependent diabetes, malnutrition, and anemia underwent a bilateral living-donor lobar lung transplantation for the treatment of end-stage lung disease secondary to cystic fibrosis. Before the operation, she had a forced expiratory volume of $20 \%$ predicted and was severely limited by her poor respiratory function. She had to use a wheelchair and required continuous supplementary oxygen. The surgery was performed with cardiopulmonary bypass support and proceeded uneventfully. The donor right and left lower lobes were implanted into the recipient right and left hemithoraces, respectively. Her chest radiograph after the operation was unremarkable, apart from mild bilateral diffuse infiltrates. On postoperative day 1 , her chest radiograph revealed a confluent opacity involving the apical segment of the right lower lobe 